\title{
The origin of the $\left\langle\mu_{\mathrm{e}}\right\rangle-M_{B}$ and Kormendy relations in dwarf elliptical galaxies
}

\author{
A. Boselli ${ }^{1}$, S. Boissier ${ }^{1}$, L. Cortese ${ }^{2}$, and G. Gavazzi ${ }^{3}$ \\ 1 Laboratoire d'Astrophysique de Marseille, UMR 6110 CNRS, 38 rue F. Joliot-Curie, 13388 Marseille France \\ e-mail: [Alessandro.Boselli; Samuel.Boissier]@oamp. fr \\ 2 School of Physics and Astronomy, Cardiff University, 5, The Parade, Cardiff CF24 3YB, UK \\ e-mail: Luca.Cortese@astro.cf.ac.uk \\ 3 Universita degli Studi di Milano-Bicocca, Piazza delle Scienze 3, 20126 Milano, Italy \\ e-mail: Giuseppe.Gavazzi@mib.infn.it
}

Received 8 February 2008 / Accepted 8 July 2008

\begin{abstract}
Aims. The present work is aimed at studying the distribution of galaxies of different types and luminosities along different structural scaling relations to see whether massive and dwarf ellipticals have been shaped by the same formation process.

Methods. This exercise is done by comparing the distribution of Virgo cluster massive and dwarf ellipticals and star forming galaxies along the $B$ band effective surface brightness and effective radius vs. absolute magnitude relations and the Kormendy relation to the predictions of models tracing the effects of ram-pressure stripping on disc galaxies entering the cluster environment and galaxy harassment.

Results. Dwarf ellipticals might have been formed from low luminosity, late-type spirals that recently entered into the cluster and lost their gas because of a ram-pressure stripping event, stopping their star formation. The perturbations induced by the abrupt decrease of the star formation activity are sufficient to modify the structural properties of disc galaxies into those of dwarf ellipticals. Galaxy harassment induces a truncation of the disc and generally an increase of the effective surface brightness of the perturbed galaxies. The lack of dynamical simulations of perturbed galaxies spanning a wide range in luminosity prevents us from deriving any firm conclusion on a possible harassment-induced origin of the low surface brightness dwarf elliptical galaxy population inhabiting the Virgo cluster.

Conclusions. Although the observed scaling relations are consistent with the idea that the distribution of elliptical galaxies along the mentioned scaling relation is due to a gradual variation with luminosity of the Sersic index $n$, the comparison with models indicates that dwarf ellipticals might have been formed by a totally different process to giant ellipticals.
\end{abstract}

Key words. galaxies: general - galaxies: structure - galaxies: formation - galaxies: evolution - galaxies: dwarf

\section{Introduction}

Scaling relations are often used as major constraints for models of galaxy formation and evolution. They can be used to trace the contribution of the different stellar components to the total luminosity of galaxies, as in the case of the color magnitude relation (Visvanathan \& Sandage 1977; Bower et al. 1992 for early-type galaxies; Tully et al. 1982; Gavazzi et al. 1996 for late-type galaxies) or to study the relationship between kinematical, structural and stellar population properties of galaxies as in the case of the Tully-Fisher relation for spirals (Tully \& Fisher 1977) and the fundamental plane for ellipticals (Dressler et al. 1987; Djorgovski \& Davis 1987). Scaling relations have been used also to compare the total gas (Boselli et al. 2002), the present or past star formation activity (Boselli et al. 2001) and the metallicity (Zaritsky et al. 1994; Bender et al. 1993) of galaxies with their luminosity or mass. The study of these different scaling relations has been crucial in showing the role of mass in the formation of galaxies (Gavazzi et al. 1996; Boselli et al. 2001), a result now generally called the downsizing effect, which is a new major constraint for hierarchical models of galaxy evolution (De Lucia et al. 2006).

The surface brightness vs. absolute magnitude and the Kormendy relation (effective surface brightness vs. effective radius; Kormendy 1985) often have been used in the literature to study the processes governing the formation of early-type galaxies. Although limited to structural properties, these two relations can be easily determined for large samples of galaxies including both giant and dwarf ellipticals. High resolution spectroscopic data, necessary for measuring velocity dispersions needed in the construction of the fundamental plane, are still relatively rare for low luminosity systems.

The study of the $B$ band surface brightness vs. absolute magnitude relation and of the Kormendy relation originally showed a strong, apparent dichotomy in the behavior of dwarf and giant ellipticals. While in dwarfs the effective or central surface brightness increases with luminosity, the opposite trend is seen in giants (e.g. Ferguson \& Binggeli 1994; Graham \& Guzman 2003). An opposite trend between giants and dwarfs also has been observed in the Kormendy relation (e.g. Kormendy 1985; Capaccioli et al. 1992). This surprising result was originally interpreted as a clear indication that dwarf ellipticals are not the low luminosity extension of giants but rather an independent class of objects. The recent study of Graham \& Guzman (2003) based on HST data however has shown that this dichotomy is due to a gradual steepening of the central radial profile with luminosity: the radial light profile of ellipticals is characterized by a Sersic law, with an index $n$ increasing from $\sim 1$ in dwarfs 
$\left(M_{B} \sim-13\right)$ to $\sim 4$ in brighter galaxies until the detection of core formation $\left(M_{B} \sim-20.5\right)$, this last probably related to the presence of a massive black hole (Faber et al. 1997; Côté et al. 2006). The different behavior of $E$ and $d E$ in the surface brightness vs. absolute magnitude relation and in the Kormendy relation is due to a gradual increase of $n$. Graham \& Guzman (2003) thus concluded that $\mathrm{d} E$ appear to be the low luminosity extension of massive ellipticals and suggested that "the mechanisms of how $\mathrm{d} E$ and $E$ galaxies collapsed to form stars are therefore expected to be similar".

Recent observational evidence and simulations, however, seem to indicate that local group dwarf spheroidals (Mayer et al. 2006), Virgo cluster dwarf ellipticals (Barazza et al. 2002; Conselice et al. 2003; van Zee et al. 2004; Mastropietro et al. 2005; Lisker et al. 2006a,b, 2007, 2008; Lisker \& Han 2008; Michielsen et al. 2008) or generally dwarf spheroidals in other clusters such as Coma (Smith et al. 2008), Perseus (Penny \& Conselice 2008) or in the SDSS (Haines et al. 2007) might be late-type galaxies recently perturbed by a hostile environment through ram-pressure stripping or galaxy harassment. In a recent work based on a multifrequency analysis of dwarf ellipticals in the Virgo cluster combined with a multizone model of the chemical and photometric evolution of galaxies, Boselli et al. (2008) have shown how several observational properties of $\mathrm{d} E$ are consistent with those of star forming discs that recently entered the cluster environment on a short time scale ( $\leq 150 \mathrm{Myr})$ losing their gas reservoir and thus abruptly stopping their star formation activity and becoming quiescent systems. It is thus natural to wonder whether the classical scaling relations such as the surface brightness vs. absolute magnitude relation and the Kormendy relation of dwarf ellipticals could be produced by environmental effects or are still a clear indication that $\mathrm{d} E$ have been formed by the same process as giant ellipticals, that the most recent models of hierarchical galaxy formation indicate as major merging (De Lucia et al. 2006).

This work is the continuation of Boselli et al. (2008), where most of the specific aspects of the comparison between models and observations were presented. The surface brightness luminosity relation has been discussed, although in a different form, in Boselli et al. (2008). Here we consider it in its general form $\left(\left\langle\mu_{\mathrm{e}}(B)\right\rangle\right.$ vs. $\left.M_{B}\right)$ and combined with the effective radius $R_{\mathrm{e}}(B)$ vs. absolute magnitude relation and the Kormendy relation. The aim of the present paper is to compare our observations to ram-pressure stripping model predictions and to the dynamical simulations of harassed galaxies in clusters. In particular we want to investigate whether the $\left\langle\mu_{\mathrm{e}}(B)\right\rangle$ vs. $M_{B}, R_{\mathrm{e}}(B)$ vs. $M_{B}$ and the Kormendy relations can be taken, as claimed by Graham \& Guzman (2003), as a clear indication that $\mathrm{d} E$ have been shaped through the same formation process as giant ellipticals. The comparison of our model predictions with observations indeed indicate that $\mathrm{d} E$ might have been formed through a totally different evolutionary process than their giant counterparts and do not result from a hierarchical assembly of matter as indicated by cosmological models.

\section{The sample}

The present analysis is based on the Virgo Cluster Catalogue (VCC) of Binggeli et al. (1985) which is an optically selected sample complete to $m_{B}<18 \mathrm{mag}$ (that for a distance of $17 \mathrm{Mpc}$ gives $M_{B} \leq-13.15$ ) at a limiting surface brightness of $\mu=$ $25.3 \mathrm{mag} \operatorname{arcsec}^{-2}$. Thanks to its proximity, the Virgo cluster is an ideal target to sample at the same time both massive and dwarf galaxies without introducing any systematic bias in the two galaxy populations due to distance uncertainties ${ }^{1}$. Galaxies analyzed in this work are all bona-fide Virgo cluster members, whose distances have been assigned following the subcluster membership criteria of Gavazzi et al. (1999) which is based on combined position and redshift data (now available for $83 \%$ of the objects). The selected sample includes a total of 1017 galaxies, 126 of which are classified as $E$ or S0, 142 as Sa-Sc, 479 as $\mathrm{d} E$ or dS0, 270 as Scd-Sd, Im, BCD, dE/Im or ? in the VCC.

\section{The data}

The $B$ band scaling relations discussed in the present communication have been reproduced using imaging photometry collected during different observational campaigns combined with data available in the literature. The major sources of $B$ band imaging data for dwarf ellipticals are Gavazzi et al. (2001, 2005), while for massive quiescent and star forming objects we use Boselli et al. (2003).

Light profile decomposition has been performed following the procedures described in Gavazzi et al. (2000). For each image we extracted the following structural parameters: the effective radius $r_{\mathrm{e}}$, defined as the radius including half of the total light, the effective surface brightness $\left\langle\mu_{\mathrm{e}}\right\rangle$, the mean surface brightness within $r_{\mathrm{e}}$ and the concentration index $C_{31}$, the ratio of the radii including $75 \%$ and $25 \%$ of the total light. Typical uncertainties are 0.1 and $0.15 \mathrm{mag}$ in $M_{B}, 0.2$ and $0.3 \mathrm{mag} \mathrm{arcsec}^{-2}$ in $\left\langle\mu_{\mathrm{e}}\right\rangle, 0.07$ and 0.12 in $\log R_{\mathrm{e}}$ and 0.2 and 0.3 in $C_{31}$ for giant and dwarf galaxies respectively. The concentration index parameter is a model independent tracer of the shape of the light profile of galaxies. The Sersic profile decomposition, probably the most appropriate for reproducing quiescent systems, is meaningless for star forming objects generally characterized by both a bulge and a disc component. In quiescent systems the concentration index is strongly related to the Sersic index $n\left(C_{31}(B)=1.49 \times n+1.33\right.$; Gavazzi et al. 2005), while in spirals values of $C_{31}(B) \leq 3$ are typical of pure exponential profiles. We thus decided to use the light concentration index parameter $C_{31}(B)$ in order to compare in a quantitative way the properties of the light profiles of early- and late-type galaxies.

Given the heterogeneous nature of the data used in the present work, we decided to limit our analysis to effective parameters, and in particular to the effective surface brightness excluding the generally used central surface brightness $\mu_{0}$ whose measure is strongly affected by the quality of the seeing (Graham \& Guzman 2003; Gavazzi et al. 2005). Furthermore we compare observations to multizone model predictions which do not implement specific treatment of the central regions and thus do not allow us to obtain an accurate estimate of the central surface brightness (see next section). Images, light profiles and structural properties of galaxies analyzed in this work can be found in the GOLDMine database (Gavazzi et al. 2003).

\section{The models}

The observed $B$ band scaling relations of dwarf ellipticals are compared to those predicted by two different models of disc galaxies in high density environments: the first one is the rampressure stripping model with subsequent quenching of the star formation activity, extensively described in Boselli et al. (2008), the second one is the galaxy harassment model of Mastropietro et al. (2005).

\footnotetext{
${ }^{1}$ Unless specified, we define as dwarf ellipticals both $\mathrm{d} E$ and dwarf spheroidals (dS0).
} 


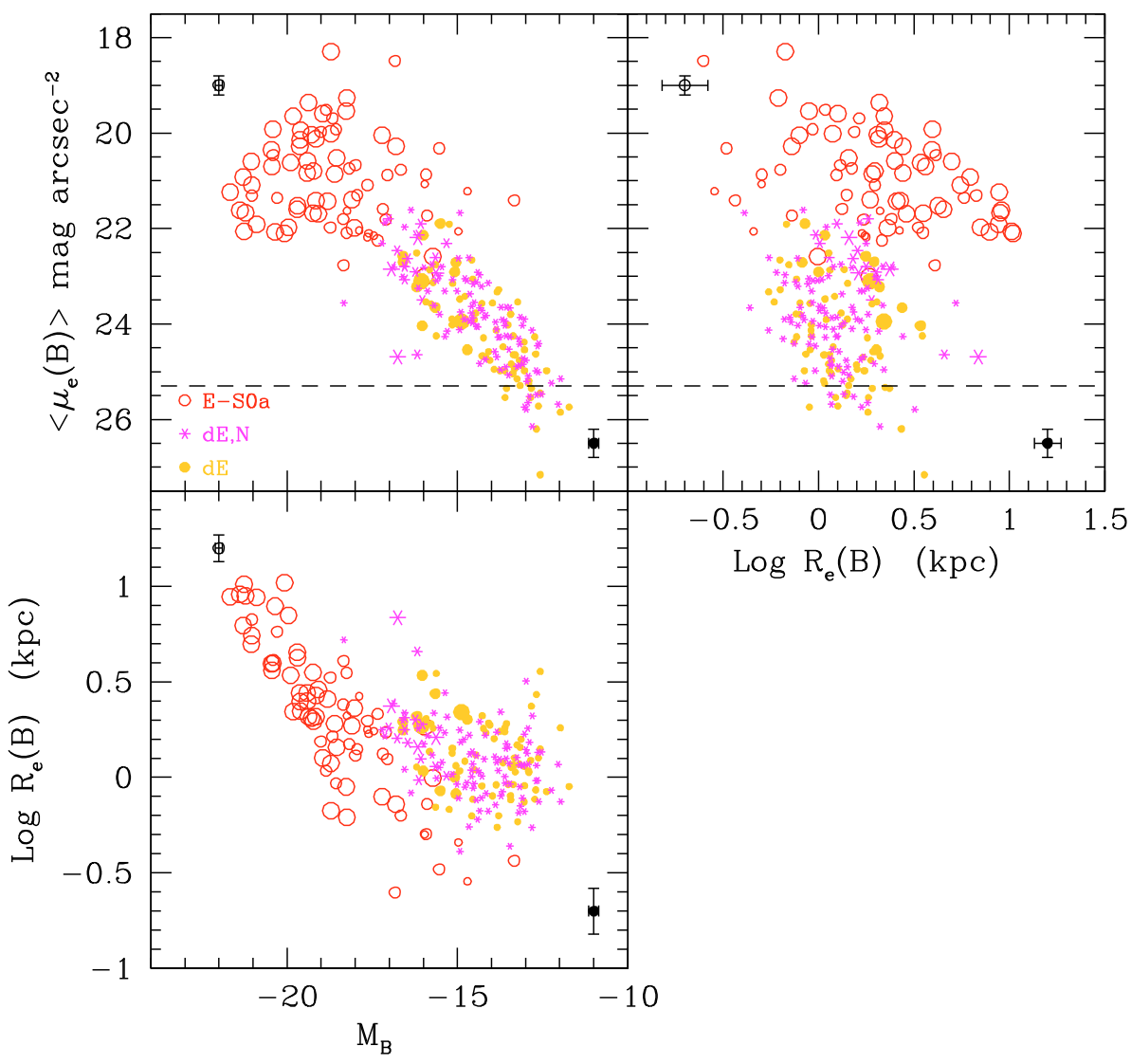

Fig. 1. The effective surface brightness $\left\langle\mu_{\mathrm{e}}(B)\right\rangle$ (upper left) and radius $R_{\mathrm{e}}(B)$ (lower left) vs. $B$ band absolute magnitude relations and the Kormendy relation $\left(\left\langle\mu_{\mathrm{e}}(B)\right\rangle\right.$ vs. radius $\left.R_{\mathrm{e}}(B)\right)$ (upper right) for early-type galaxies: red, open circles are for ellipticals and lenticulars $(E-S 0-$ $\mathrm{S} 0 \mathrm{a})$, magenta asterisks for nucleated dwarf ellipticals $(\mathrm{d} E, N)$, orange, filled dots for $\mathrm{d} E$. Sizes of the symbols have been chosen according to the light concentration index: large for $C_{31}(B)>5$; medium for $3.5<C_{31}(B) \leq 5$ and small for $C_{31}(B) \leq 3.5$. The horizontal dashed line gives the VCC surface brightness detection limit (25.3 mag $\left.\operatorname{arcsec}^{-2}\right)$. Typical error bars for giants and dwarfs are given.
The evolution of galaxies in a ram-pressure stripping model is traced using the multi-zone chemical and spectrophotometric models of Boissier \& Prantzos (2000), updated with an empirically-determined star formation law (Boissier et al. 2003a) relating the star formation rate to the total-gas surface densities, and modified to simulate a ram-pressure stripping event induced by the interaction with the cluster IGM. The free parameters in this grid of models are the spin parameter, $\lambda$ and the rotational velocity, $V_{C}$. These two parameters are theoretical quantities, although $V_{\mathrm{C}}$ should be similar to the asymptotic value of the rotation curve at large radii. The spin parameter is a dimensionless measure of the specific angular momentum (defined in e.g. Mo et al. 1998). Its value in spirals ranges typically from $\sim 0.02$ for relatively compact galaxies and $\gtrsim 0.07$ corresponding to low surface brightness galaxies (Boissier et al. $2003 \mathrm{~b}$ ), although $\sim 50 \%$ of the galaxies (including LSB) have values in the range $0.3 \leq \lambda \leq 0.6$. In the models of Boissier \& Prantzos (2000) the total mass varies as $V_{\mathrm{C}}^{3}$, the scale-length as $\lambda \times V_{\mathrm{C}}$. Star formation histories depend on the infall timescales, which are a function of $V_{\mathrm{C}}$, so that roughly speaking, $V_{\mathrm{C}}$ controls the stellar mass accumulated during the history of the galaxy, and $\lambda$ its radial distribution.

The ram-pressure exerted by the hot and dense IGM (Gunn $\&$ Gott 1972) on galaxies crossing the cluster with velocities of $\sim 1000 \mathrm{~km} \mathrm{~s}^{-1}$ can easily remove the galaxy ISM, in particular the atomic hydrogen located outside the optical disc, quenching their star formation activity (Boselli \& Gavazzi 2006). The rampressure stripping event is simulated here by assuming a gas-loss rate inversely proportional to the potential of the galaxy, with an efficiency depending on the IGM gas density radial profile of the Virgo cluster given by Vollmer et al. (2001).

For simplicity we will consider here only a ram-pressure stripping model with efficiency $\epsilon_{0}=1.2 M_{\odot} \mathrm{kpc}^{-2} \mathrm{yr}^{-1}$ (see
Boselli et al. 2008 for details). This efficiency, typical for the gas-stripped spiral galaxies in Virgo, can be taken as the average value needed to produce the dwarf elliptical galaxy population through ram-pressure stripping. We also consider only rampressure stripping since it was previously shown that starvation does not seem to reproduce the observed properties of stripped galaxies (Boselli et al. 2006, 2008).

The harassment model is taken from Mastropietro et al. (2005): this is based on N-body simulations of disc galaxies within a $\Lambda \mathrm{CDM}$ cluster with $10^{7}$ particles, where the hierarchical growth and galaxy harassment are simulated selfconsistently. These simulations indicate that most of the galaxies undergo major structural modifications even at the outskirts of the cluster, with a large fraction of them transforming from late-type rotating systems into dwarf spheroidal hot systems. These N-body simulations are well adapted for comparison since they have been defined to reproduce the evolution of a relatively low-luminosity late-type galaxy (absolute magnitude $M_{B}=-17.08$ ) in a cluster of mass similar to Virgo. Total masses and effective radii for 13 perturbed simulated galaxies have been kindly provided by Mastropietro, and have been transformed into $B$ band luminosities assuming a constant $M / L_{B}$ ratio of 6 (and $M / L_{B}=4.5$ for the unperturbed model), as indicated in Mastropietro et al. (2005).

\section{The scaling relations}

The available set of data allows us to reconstruct the effective surface brightness $\left\langle\mu_{\mathrm{e}}(B)\right\rangle$ and radius $R_{\mathrm{e}}(B)$ vs. $B$ band absolute magnitude relations and the Kormendy relation $\left(\left\langle\mu_{\mathrm{e}}(B)\right\rangle\right.$ vs. radius $\left.R_{\mathrm{e}}(B)\right)$ for both early (E, S0-S0a, $\mathrm{d} E$-dSO) and latetype (Spirals, Im, BCD) galaxies in the Virgo cluster (respectively in Figs. 1 and 2). The surface brightness vs. absolute 


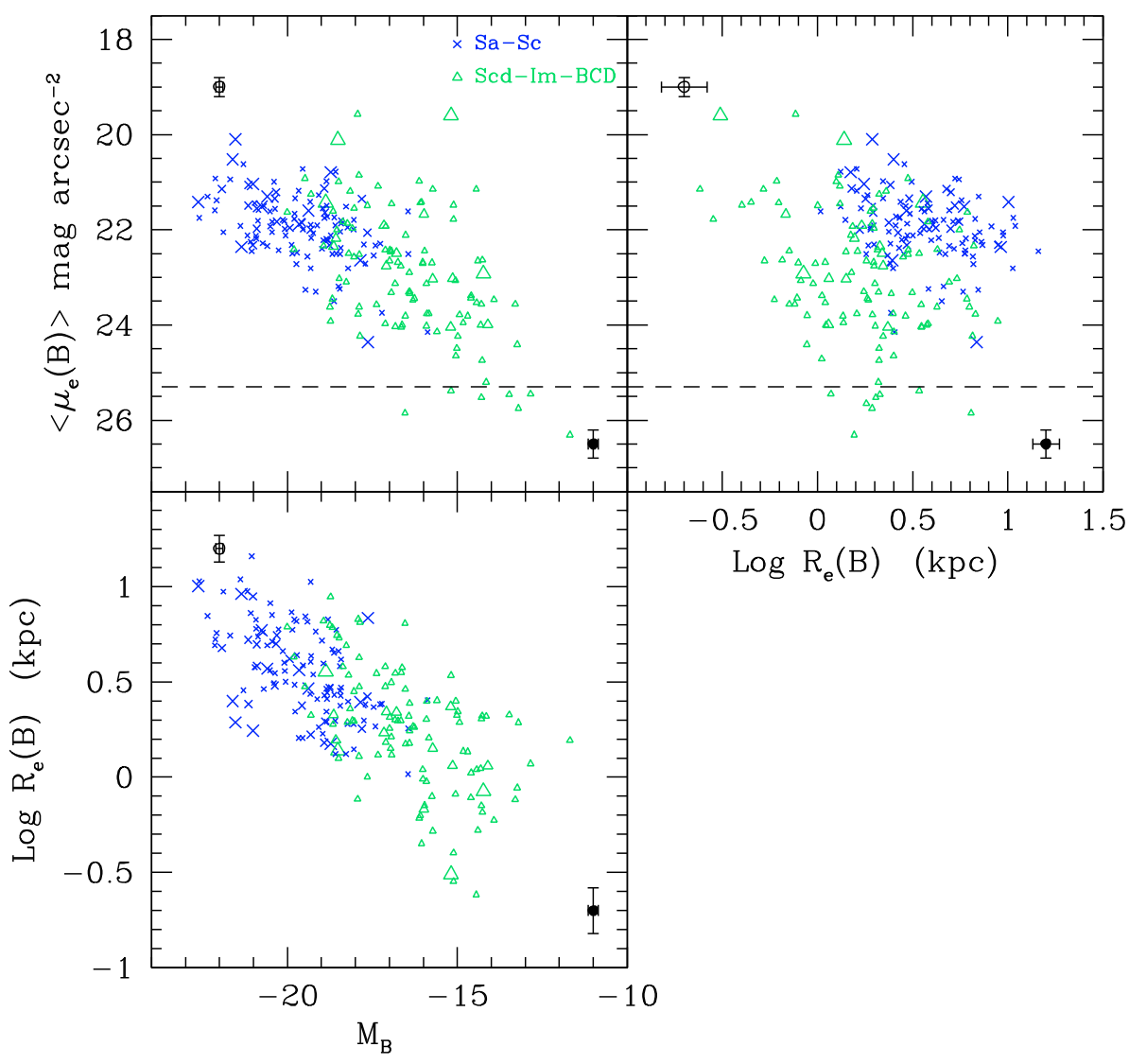

Fig. 2. The effective surface brightness $\left\langle\mu_{\mathrm{e}}(B)\right\rangle$ (upper left) and radius $R_{\mathrm{e}}(B)$ (lower left) vs. $B$ band absolute magnitude relations and the Kormendy relation $\left(\left\langle\mu_{\mathrm{e}}(B)\right\rangle\right.$ vs. radius $R_{\mathrm{e}}(B)$ ) (upper right) for late-type galaxies (blue crosses for Sa-Sc, green open triangles for ScdIm-BCD). Sizes of the symbols have been chosen according to the light concentration index: large for $C_{31}(B)>5$; medium for $3.5<$ $C_{31}(B) \leq 5$ and small for $C_{31}(B) \leq 3.5$.

magnitude relation of quiescent systems shows the well known different behavior of massive and dwarf galaxies, with the surface brightness increasing with luminosity in dwarfs and decreasing in giants (e.g. Ferguson \& Binggeli 1994; Graham \& Guzman 2003). The effective radius increases with luminosity in giant ellipticals and lenticulars, while it is almost constant (albeit with a huge scatter) in dwarfs with absolute magnitudes $M_{B}>-18$. A substantial difference between giants and dwarfs can be seen in the Kormendy relation, where a significant dichotomy in the effective surface brightness vs. radius distribution is is present between $\mathrm{d} E$ and E-S0-SOa. Considering the strong relationship between the Sersic index $n$ and the concentration index $C_{31}(B)$ shown by Gavazzi et al. (2005; see their Fig. 12), the size variation of the adopted symbols along the relations is consistent with the apparent dichotomy in the structural properties of dwarf and giants is due to an increase of the Sersic index $n$ with luminosity (Graham \& Guzman 2003).

The surface brightness vs. absolute magnitude relation of star forming systems is similar to that of dwarf ellipticals, with the surface brightness increasing with luminosity (see Fig. 2), although with a flatter slope and a significant larger scatter. The effective radius increases with absolute magnitude in star forming galaxies for all luminosities but with a flatter slope than in giant ellipticals. The Kormendy relation of early-type spirals (Sa-Sc) and ellipticals and that of late-type spirals and irregulars (ScdIm-BCD) and $\mathrm{d} E$ respectively are basically similar, although the difference in between the two quiescent populations seems more pronounced than for the star forming systems.

The relationship between the absolute magnitude, the effective surface brightness and radii and the concentration index are given in Fig. 3. The large majority of both nucleated and non dwarf ellipticals have concentration indices $\sim 3$, thus Sersic indices $n \sim 1$. All have effective surface brightnesses $\geq 21.5 \mathrm{mag} \operatorname{arcsec}^{-2}$ and most of them have effective radii in the range $0.5 \leq R_{\mathrm{e}}(B) \leq 2 \mathrm{kpc}$. Giant ellipticals and lenticulars have light concentration indices $C_{31}(B)>3$ (thus Sersic indices $n>1$ ), effective surface brightnesses brighter than $23 \mathrm{mag} \operatorname{arcsec}^{-2}$ and span a larger range in effective radii than $\mathrm{d} E\left(0.3 \leq R_{\mathrm{e}}(B) \leq 10 \mathrm{kpc}\right)$. Low luminosity star forming and quiescent galaxies $\left(M_{B} \geq-18\right)$ all have small $\left(C_{31}(B) \sim 3\right)$ light concentration indices. The light concentration index of massive galaxies is systematically higher in ellipticals and lenticulars $\left(C_{31}(B) \geq 5\right.$ for $\left.M_{B} \leq-20\right)$ than in spirals $\left(C_{31}(B) \leq 6\right)$. Late-type galaxies share the same effective surface brightness and radius vs. $C_{31}(B)$ relationships than dwarf ellipticals (both populations are dominated by roughly exponential profiles), although star forming systems have on average slightly higher surface brightnesses and larger radii than dwarf ellipticals.

These scaling relations are consistent with those observed in other clusters: we notice however that quiescent dwarfs in Virgo have slightly larger (but consistent) mean effective radii with a significantly more dispersed distribution than Antlia (Smith Castelli et al. 2008) and Coma (Graham \& Guzman 2003) dwarfs, being $R_{\mathrm{e}}(B)=1.41 \pm 0.79 \mathrm{kpc}$ for all the $\mathrm{d} E$ of the sample, $R_{\mathrm{e}}(B)=1.40 \pm 0.87 \mathrm{kpc}$ for the nucleated and $R_{\mathrm{e}}(B)=1.43 \pm 0.66 \mathrm{kpc}$ for the non nucleated ones. This difference might be due to large statistical uncertainties in the Antlia (36 galaxies) and Coma (18 galaxies) samples with respect to Virgo (187 objects) or to a slightly different sampled range in luminosity and surface brightness.

To test possible effects related to the galaxy position within Virgo we compared the $\left\langle\mu_{\mathrm{e}}(B)\right\rangle$ vs. $M_{B}, R_{\mathrm{e}}(B)$ vs. $M_{B}$ and the Kormendy relations for galaxies located within and outside half the cluster virial radius $\left(2.82^{\circ}, 0.84 \mathrm{Mpc}\right)$. No significant differences have been observed between galaxies located close to the core or at the periphery of the cluster. 

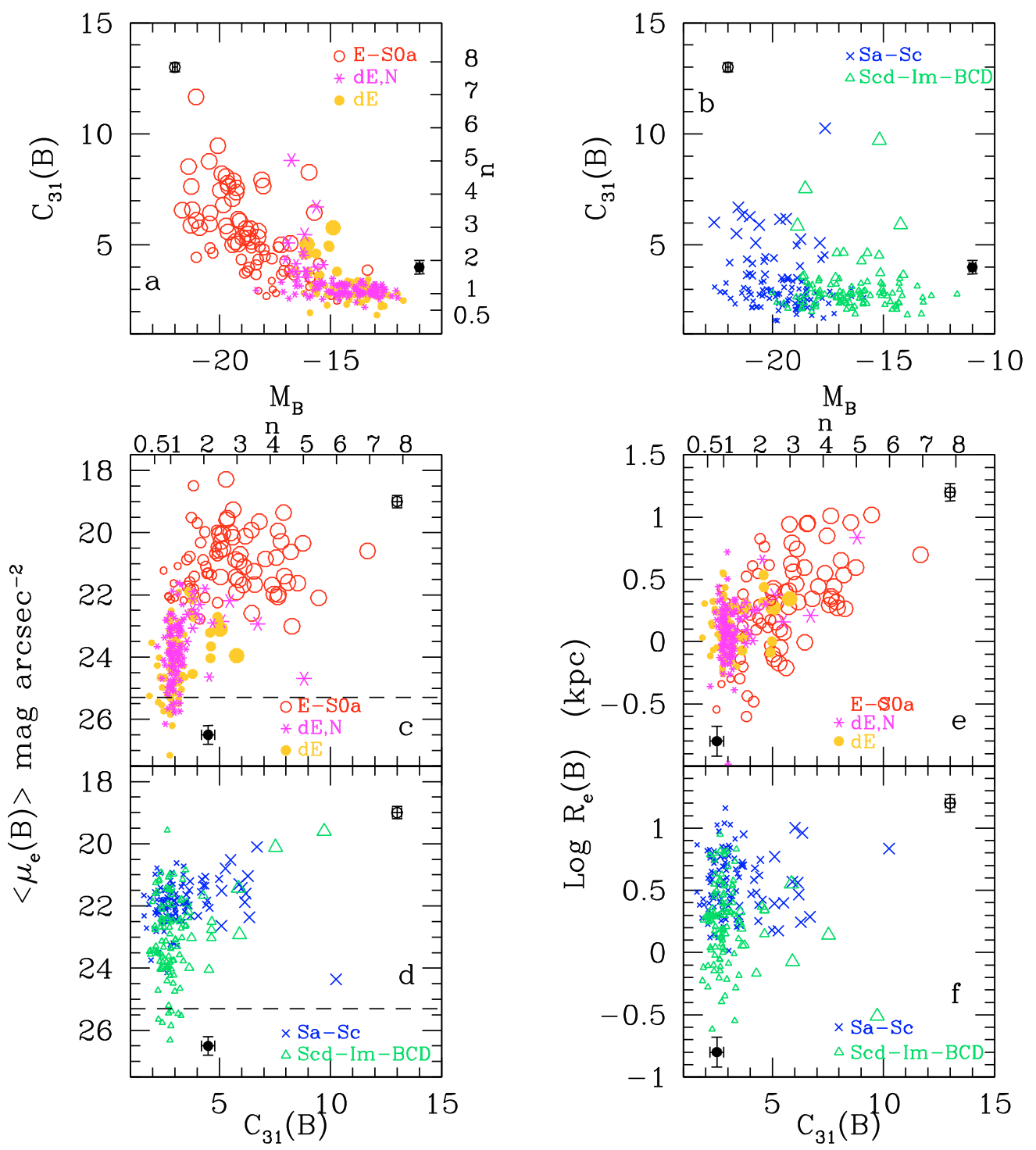

Fig. 3. The relationship between the concentration index $C_{31}(B)$ and the absolute magnitude $M_{B}$ for early-type a) and for late-type galaxies b), the effective surface brightness $\left\langle\mu_{\mathrm{e}}(B)\right\rangle$ and the concentration index $C_{31}(B)$ for early-type c) and for late-type galaxies d) and the effective radius $R_{\mathrm{e}}(B)$ and the concentration index $C_{31}(B)$ for early-type e) and for late-type galaxies f). Symbols are as in Figs. 1 and 2. The corresponding Sersic index $n$ valid for early-type galaxies has been determined from the relation $C_{31}(B)=1.49 \times n+1.33$ (Gavazzi et al. 2005).

\section{Comparison with model predictions}

Ram-pressure models show how different structural parameters change once discs stop their star formation activity because of gas removal. Model predictions are compared to observations in Fig. 5. Since models represent disc galaxies without bulges, they cannot be used to predict the variation of the light concentration index which is a bulge sensitive parameter. We thus first compare model predictions to the scaling relation determined for galaxies with roughly exponential profiles $\left(C_{31}(B) \leq 3.5\right.$, small symbols in Fig. 5).

The observed effective surface brightness vs. absolute magnitude relation (upper left panel in Fig. 5) shows a weak decrease of the effective surface brightness with decreasing luminosity in bright objects (mostly spirals), and a steeper relation at lower luminosities (dwarf ellipticals). The models indicate that this trend can be reproduced by stopping the star formation activity of star forming discs. While the perturbations in massive galaxies $\left(V_{\mathrm{C}}=220 \mathrm{~km} \mathrm{~s}^{-1}\right)$ are minor, in low luminosity objects $\left(V_{\mathrm{C}} \leq 70 \mathrm{~km} \mathrm{~s}^{-1}\right)$ the surface brightness and the absolute magnitude are significantly reduced with respect to that of a similar unperturbed object.

Models also predict the observed variation of the effective radius with absolute magnitude, with a decrease of $R_{\mathrm{e}}(B)$ with decreasing luminosity down to $M_{B} \sim-17$ (spirals) and a roughly constant value at lower luminosities, as indeed observed in dwarf ellipticals. Model predictions indicate that the Kormendy relation $\left(\left\langle\mu_{\mathrm{e}}(B)\right\rangle\right.$ vs. radius $\left.R_{\mathrm{e}}(B)\right)$ of dwarf ellipticals can result from the transformation of star forming galaxies once their activity is stopped because of gas removal after a ram-pressure stripping event. A large scatter in the effective surface brightness $(\sim 1.5 \mathrm{mag})$ and radius $(0.4 \mathrm{in}$ dex $)$ are predicted by models if we consider that the spin parameter is expected to vary in normal galaxies in the range $0.03 \leq \lambda \leq 0.06$ (see Fig. 4). More extreme values of $\lambda$, although possible, are expected to be rare. The observed scatter in the $R_{\mathrm{e}}(B)$ and $\left\langle\mu_{\mathrm{e}}(B)\right\rangle$ parameters in Fig. 5 can thus be ascribed to the distribution in the spin parameter expected in normal, disc galaxies. Among spiral galaxies, the most discrepant from models of unperturbed objects are, as expected, those with prominent bulges $\left(C_{31}(B)>3.5\right.$, large and medium 


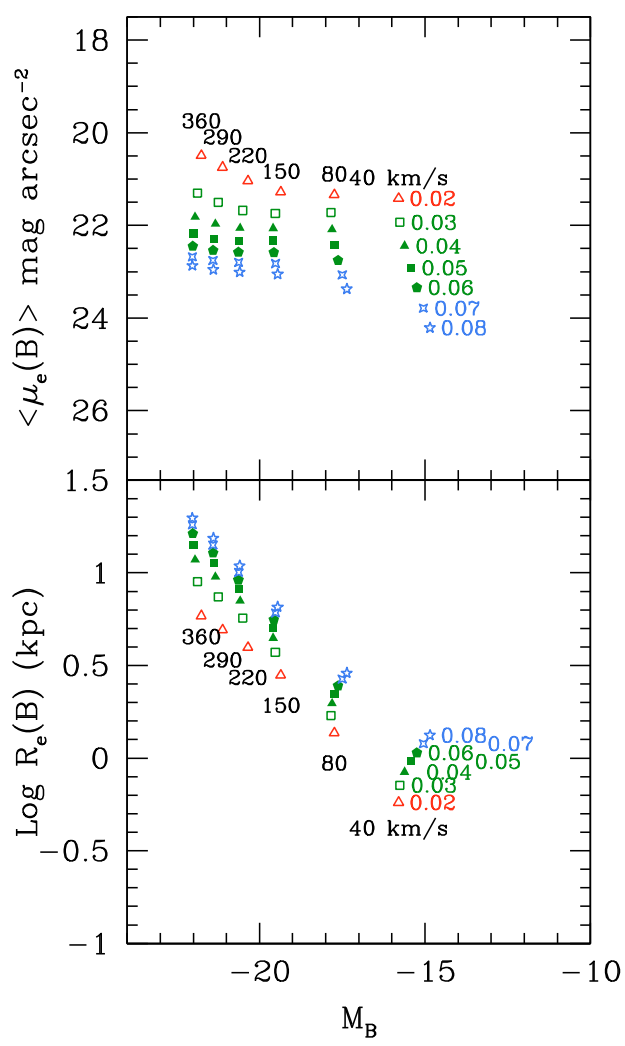

Fig. 4. The model $B$ band effective surface brightness (upper panel) and radius (lower panel) as a function of the absolute magnitude for different spin parameters (from 0.02 to 0.08 ) and different velocities (from 40 to $360 \mathrm{~km} \mathrm{~s}^{-1}$ ) in unperturbed, disc galaxies. This indicates the scatter expected in various relationships due to the distribution of the spin parameter.

blue crosses and green triangles), this component not being considered by our models.

Figure 6 shows the effects of ram-pressure stripping (filled black squares indicate unperturbed galaxies of different rotational velocity, black dashed lines the evolution after a rampressure stripping event) and galaxy harassment (the red empty pentagon shows the unperturbed galaxy, blue filled pentagons the 13 different perturbed models) with respect to the observations, here indicated by continuous, dotted and dashed contours for the distribution of $\mathrm{d} E-\mathrm{d} E, N$ (orange), spiral, Im and $\mathrm{BCD}$ (green), ellipticals and lenticulars (red) respectively. The comparison of the observed scaling relations with the dynamical simulations of Mastropietro et al. (2005) indicate that harassed galaxies are more compact and have comparable or higher surface brightness than unperturbed objects (see Fig. 6), probably because of the formation of bars (Mastropietro et al. 2005). Their absolute magnitude decreases after the interaction because of mass loss. Dynamical interactions thus have significantly different effects to those induced by a ram-pressure stripping event.

Harassed galaxies have structural properties in between those of low-luminosity and and massive ellipticals. It is however difficult to state whether harassed galaxies fail to reproduce the low-luminosity, low-surface brightness dwarf ellipticals at the origin of the $\left\langle\mu_{\mathrm{e}}(B)\right\rangle$ vs. $M_{B}, R_{\mathrm{e}}(B)$ vs. $M_{B}$ and the Kormendy relations just because dynamical simulations are only available for an intermediate mass disc galaxy.

The structural properties of giant ellipticals and lenticulars (red open circles) are significantly different from those of massive spirals (blue crosses) and, as expected, cannot be produced by a simple quenching of the star formation activity of spirals after a ram-pressure stripping event. The dynamical simulations of Mastropietro et al. (2005), available only for a relatively low-mass late-type galaxy, prevent us from seeing whether the enhanced surface brightness of early-type galaxies, and in particular of S0s, with respect to that of late-types of similar luminosity, could result from galaxy harassment. To answer this important question it would be necessary to extend the simulation of Mastropietro et al. (2005) to cover the dynamic range in stellar mass of the late-type galaxy population of the Virgo cluster.

We can thus conclude that most of the dwarf ellipticals have structural properties consistent with being ram-pressure gas stripped, low luminosity star forming galaxies, as stated in Boselli et al. (2008). The lack of any observed trend in the scaling relations with the clustercentric distance is in agreement with this view since the gas stripping phenomenon and the subsequent stopping of the star formation activity acts on relatively short times scales ( $\leq 150 \mathrm{Myr}$, Boselli et al. 2008) if compared to the cluster crossing time, which in Virgo is of the order of $1.7 \mathrm{Gyr}$. It is thus almost impossible to observe objects in their Im/BCD$\mathrm{d} E$ transitionary phase. The observed morphology-segregation effect in dwarfs (Ferguson \& Binggeli 1994) is indeed consistent with this picture.

\section{Discussion and conclusion}

The analysis presented so far is fully consistent with the idea that the origin of the different behavior of dwarf and massive early-type galaxies along the $B$ band effective surface brightness and effective radius vs. absolute magnitude relations and along the Kormendy relation often described in the literature is closely related to the shape of their radial light profiles, as suggested by Graham \& Guzman (2003). We indeed observe that the effective surface brightness decreases (and the effective radius increases) with luminosity only in early-type galaxies with high concentration indices or high Sersic indices $n$, while it increases (it is almost constant) whenever $C_{31}(B)<5$ or $n<2.5$. Massive $\left(M_{B} \leq-20\right)$ ellipticals and lenticulars all have $C_{31}(B) \geq 5$, while quiescent dwarfs $\left(M_{B} \geq-18\right)$ have $C_{31}(B) \sim 3$ consistent with a roughly exponential profile.

In late-type galaxies the effective surface brightness and the effective radius monotonically increase with luminosity, but with flatter slopes and larger scatters than in low luminosity and massive ellipticals respectively. They all have $C_{31}(B) \sim \leq 6$, with $C_{31}(B) \sim 3$ in $\sim 70 \%$ of the massive objects.

The comparison with models shows that the distribution of quiescent galaxies along the aforementioned scaling relations cannot be unequivocally taken as a proof of a similar formation process for dwarf and giants, as stated by Graham \& Guzman (2003). The lack of dynamical simulations for the whole range in luminosity of the disc galaxy population prevents us from determining whether the scaling relations observed in $E-\mathrm{d} E$ galaxies discussed here can be the result of the harassment suffered by spirals entering the cluster.

The observed relations between the effective surface brightness, effective radius and absolute magnitude of low luminosity ellipticals are consistent with their formation after a recent and rapid decrease of the star formation activity of low luminosity spirals recently entering the cluster medium and swept of their gas by a ram-pressure stripping event, as claimed by Boselli et al. (2008). This evolutionary path is thus significantly different than that followed by massive ellipticals for which hierarchical models of galaxy formation predict they formed most of their stars at 

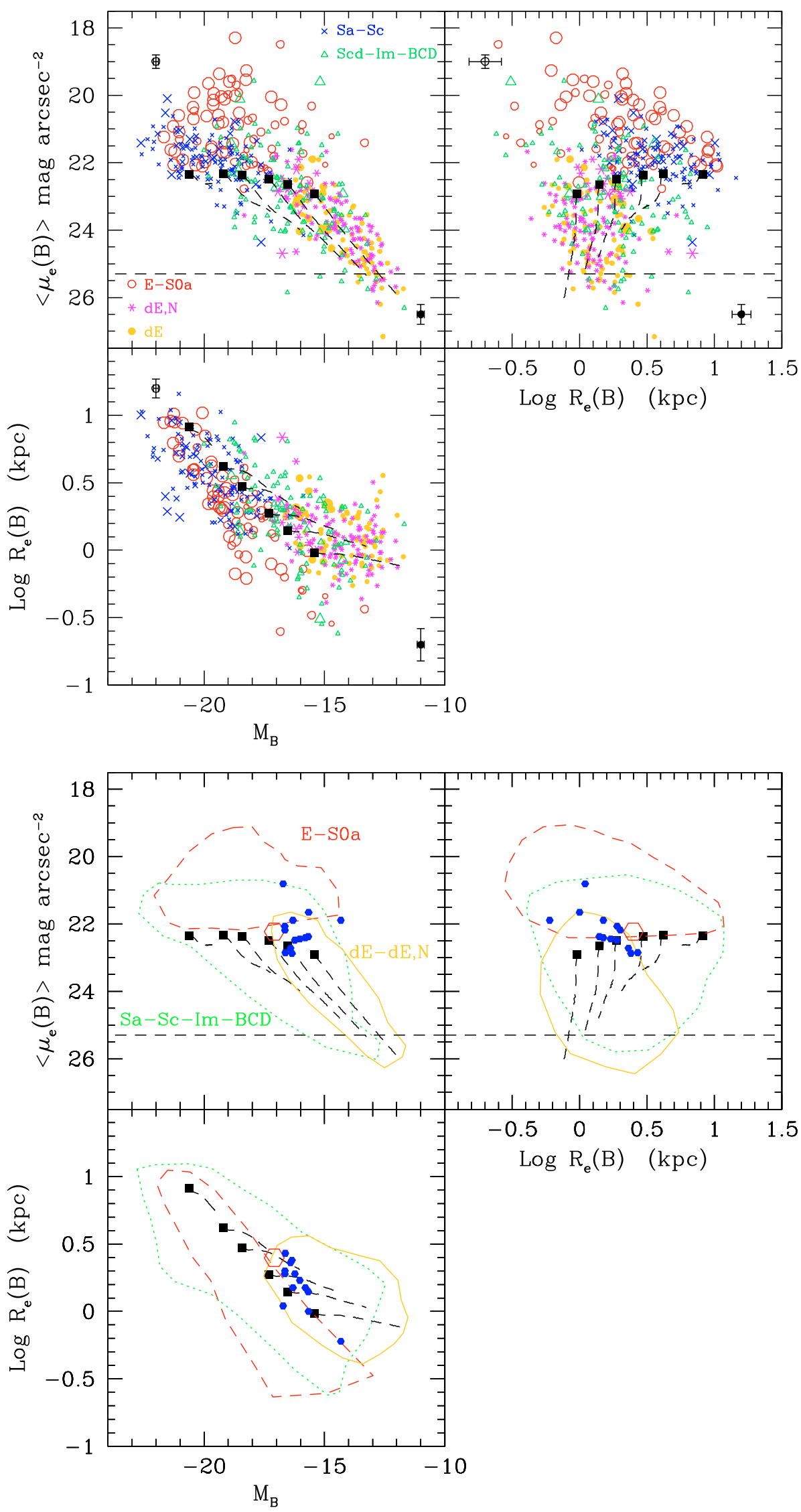

Fig. 5. Comparison of the model predictions to the scaling relations for all sample galaxies. Symbols are as in Figs. 1 and 2. Model predictions for unperturbed galaxies of spin parameter $\lambda=0.05$ and $V_{\mathrm{C}}=40,55,70,100,130$ and $220 \mathrm{~km} \mathrm{~s}^{-1}$ are indicated with filled black squares. Model predictions for a ram-pressure stripping event at different epochs are indicated by black dashed line.

Fig. 6. Comparison of our ram-pressure stripping model predictions and those predicted by Mastropietro et al. (2005) for galaxy harassment to the scaling relations for all sample galaxies. Our ram-pressure model predictions for unperturbed galaxies of spin parameter $\lambda=0.05$ and $V_{\mathrm{C}}=40,55,70,100$, 130 and $220 \mathrm{~km} \mathrm{~s}^{-1}$ are indicated with filled black squares, and their evolution after a rampressure stripping event by black dashed lines. The unperturbed galaxy for the models of Mastropietro et al. (2005) is indicated by a large, red hexagon while the simulated harassed galaxies by small, blue filled hexagons. Continuum, dotted and dashed contours indicate the distribution of $\mathrm{d} E-\mathrm{d} E, N$ (orange), spiral, Im and BCD (green), ellipticals and lenticulars (red) galaxies as determined by eye from our own observations. early epochs $(z \geq 2)$ and later assembled through major merging events (De Lucia et al. 2006). A different evolution of massive and dwarf ellipticals is also confirmed by the analysis of their spectro-photometric properties and of their $[\alpha / \mathrm{Fe}]$ ratios, both indicating a rapid formation of the bulk of the stellar population in the early universe in massive ellipticals with a rather continuous 
and moderate star formation activity for dwarf systems (Thomas et al. 2005; Renzini 2006).

The present work is a further indication that the interpretation of single scaling relations in terms of evolution is not always straightforward but rather needs to be included in a complete and coherent study combining multifrequency observations with model predictions.

Acknowledgements. This research has made use of the NASA/IPAC Extragalactic Database (NED) which is operated by the Jet Propulsion Laboratory, California Institute of Technology, under contract with the National Aeronautics and Space Administration, and of the GOLD Mine database. We are grateful to C. Mastropietro who provided us with the results of their N-body dynamical simulations, and the anonymous referee for precious comments and suggestions which helped to improve the quality of the manuscript.

\section{References}

Barazza, F., Binggeli, B., \& Jerjen, H. 2002, A\&A, 391, 823

Bender, R., Burstein, D., \& Faber, S. 1993, ApJ, 411, 153

Binggeli, B., Sandage, A., \& Tammann, G. 1985, AJ, 90, 1681

Boissier, S., \& Prantzos, N. 2000, MNRAS, 312, 398

Boissier, S., Prantzos, N., Boselli, A., \& Gavazzi, G. 2003a, MNRAS, 346, 1215

Boissier, S., Monnier Ragaigne, D., Prantzos, N., et al. 2003b, MNRAS, 343, 653

Boselli, A., \& Gavazzi, G. 2006, PASP, 118, 517

Boselli, A., Gavazzi, G., Donas, J., \& Scodeggio, M. 2001, AJ, 121, 753

Boselli, A., Lequeux, J., \& Gavazzi, G. 2002, A\&A, 384, 33

Boselli, A., Gavazzi, G., \& Sanvito, G. 2003, A\&A, 402, 37

Boselli, A., Boissier, S., Cortese, L., et al. 2006, ApJ, 651, 811

Boselli, A., Boissier, S., Cortese, L., \& Gavazzi, G. 2008, ApJ, 674, 742

Bower, R. G., Lucey, J. R., \& Ellis, R. S. 1992, MNRAS, 254, 601

Capaccioli, M., Caon, N., \& D’Onofrio, M. 1992, MNRAS, 259, 323

Conselice, C., O’Neil, K., Gallagher, J., \& Wyse, R. 2003, ApJ, 591, 167

Côté, P., Piatek, S., Ferrarese, L., et al. 2006, ApJS, 165, 57

De Lucia, G., Springel, V., White, S., Croton, D., \& Kauffmann, G. 2006, MNRAS, 366, 499
Djorgovski, S., \& Davis, M. 1987, ApJ, 313, 59

Dressler, A., Lynden-Bell, D., Burstein, D., et al. 1987, ApJ, 313, 42

Faber, S., Tremaine, S., Ajhar, E., et al. 1997, AJ, 114, 1771

Ferguson, H., \& Binggeli, B. 1994, A\&A Rev., 6, 67

Gavazzi, G., Pierini, D., \& Boselli, A. 1996, A\&A, 312, 397

Gavazzi, G., Boselli, A., Scodeggio, M., Pierini, D., \& Belsole, E. 1999, MNRAS, 304, 595

Gavazzi, G., Franzetti, P., Scodeggio, M., Boselli, A., \& Pierini, D. 2000, A\&A, 361,863

Gavazzi, G., Zibetti, S., Boselli, A., et al. 2001, A\&A, 372, 29

Gavazzi, G., Boselli, A., Donati, A., Franzetti, P., \& Scodeggio, M. 2003, A\&A, 400,451

Gavazzi, G., Donati, A., Cuccati, O., et al. 2005, A\&A, 430, 411

Graham, A., \& Guzman, R. 2003, AJ, 125, 2936

Gunn, J. E., \& Gott, J. R. I. 1972, ApJ, 176, 1

Haines, C., Gargiuolo, A., La Barbera, F., et al. 2007, MNRAS, 381, 7

Haines, C., Gargiuolo, A., \& Merluzzi, P. 2008, MNRAS, 385, 1201

Kormendy, J. 1985, ApJ, 432, L63

Kormendy, J., \& Kennicutt, R. 2004, ARA\&A, 42, 603

Lisker, T., \& Han, Z. 2008, ApJ, 680, L1042

Lisker, T., Grebel, E., \& Binggeli, B. 2006a, AJ, 132, 497

Lisker, T., Glatt, K., Westera, P., \& Grebel, E. 2006b, AJ, 132, 2432

Lisker, T., Grebel, E., Binggeli, B., \& Glatt, K. 2007, ApJ, 660, 1186

Lisker, T., Grebel, E., \& Binggeli, B. 2008, AJ, 135, 380

Mayer, L., Mastropietro, C., Wadsley, J., Stadel, J., \& Moore, B. 2006, MNRAS, 369,1021

Mastropietro, C., Moore, B., Mayer, L., et al. 2005, MNRAS, 364, 607

Michielsen, D., Boselli, A., Conselice, C., et al. 2008, MNRAS, 385, 1374

Mo, H. J., Mao, S., \& White, S. D. M. 1998, MNRAS, 295, 319

Penny, S., \& Conselice, C. 2008, MNRAS, 383, 247

Renzini, A. 2006, ARA\&A, 44, 141

Smith, R., Marzke, R., Hornschemeier, A., et al. 2008, MNRAS, 386, L96 Smith Castelli, A., Bassino, L., Richtler, T., et al. 2008, MNRAS, 386, 2311

Thomas, D., Maraston, C., Bender, R., \& Mendes de Oliveira, C. 2005, ApJ, 621, 673

Tully, B., \& Fisher, J. 1977, A\&A, 54, 661

Tully, B., Mould, J., \& Aaronson, M. 1982, ApJ, 257, 527

Van Zee, L., Skillman, E., \& Haynes, M. 2004, AJ, 128, 121

Visvanathan, N., \& Sandage, A. 1977, ApJ, 216, 214

Vollmer, B., Cayatte, V., Balkowski, C., \& Duschl, W. 2001, ApJ, 561, 708

Zaritsky, D., Kennicutt, R., \& Huchra, J. 1994, ApJ, 420, 87 